\title{
Notas para problematizar la ética de sí en la educación del cuerpo: la clase de
}

\section{Educación Física como territorio de disputas de género}

\author{
RODRÍGUEZ, Norma Beatriz (Buenos Aires, Argentina) $)^{1 *}$ \\ BUSTOS, Liliana (Buenos Aires, Argentina) $)^{2 *}$ \\ 1 Universidad Nacional de La Plata, Departamento de Educación Física, \\ Facultad de Humanidades y Ciencias de la Educación \\ Escuela Remedios de Escalada de San Martín, Facultad de Humanidades y Ciencias de la Educación \\ ORCID ID: https://orcid.org/0000-0003-0250-1477* \\ ORCID ID: https://orcid.org/0000-0001-9766-2614**
}

\begin{abstract}
Resumen
La creación de la escuela moderna a finales del siglo XIX trajo consigo la formación de una disciplina ocupada de educar el cuerpo. Fue en ese momento constitutivo en donde se delimitaron las prácticas corporales para los hombres: ejercicios militares, fuerza, virilidad, posturas; mientras que para las mujeres se esperaban: perfección de la feminidad, danza, gimnasia rítmica. De la perpetuación de esos estereotipos en la clase de Educación Física deriva la hipótesis de esta investigación: la influencia directa y condicionada de las prácticas culturales en relación a la expresión de género queda reducida al análisis respecto de la condición de sexo leído en clave de lo que es biológicamente atribuido. Como marco teórico, se recurrió a los aportes de la Teoría Queer y, por otro lado, se analizaron las normativas vigentes para el Sistema Educativo Argentino tomando especial referencia en la Ley de Educación Sexual Integral de la Argentina. El trabajo de campo se realizó durante el año 2018 en dos escuelas de nivel primario de administración pública situadas en las ciudades de Almafuerte y Rio Tercero de la provincia de Córdoba, Argentina.
\end{abstract}

Palabras clave

Educación Física. Género. Construcción cultural. Educación del cuerpo.

\section{Notas para problematizar a ética de si na educação do corpo: as aulas de} Educação Física como território de disputas de gênero

\begin{abstract}
Resumo
A criação da escola moderna aos finais do século XIX trouxe consigo uma disciplina que se ocupou da educação dos corpos. Foi nesse momento constitutivo em que se delimitaram as práticas corporais para homens: exercícios militares, força, virilidade, posturas; ao mesmo tempo que, para a educação dos corpos das mulheres, se esperavam: perfeição da feminilidade, dança, ginástica rítmica. É da perpetuação desses estereótipos de comportamentos nas aulas de Educação Física como conteúdo escolar que deriva a hipótese desta pesquisa: as aulas de Educação Física influenciam e condicionam diretamente as práticas culturais em relação à expressão de gênero reduzindo a condição de sexo ao que está inscrito na biologia do corpo. Como marco teórico, recorreu-se aos aportes teóricos da Teoria Queer e, por outro lado, analisaram-se as normativas vigentes para o Sistema Educativo Argentino tomando especialmente como referência a Lei de Educação Sexual
\end{abstract}


Integral da Argentina. O trabalho de campo se realizou durante o ano de 2018 em duas escolas de nível primário de administração públicas situadas nas cidades de Almafuerte e Rio Terceiro do estado de Córdoba, Argentina.

\title{
Palavras-chave
}

Educação Física. Gênero. Construção cultural. Educação do corpo.

\author{
Notes for problematizing the ethics of self in education: \\ the class of Physical Education as a territory for gender disputes
}

\begin{abstract}
The creation of the modern school to finals of XIX century brought the formation of a discipline to educate the body. It was in that constitutive moment where practices were delimited for men: military exercises, force, virility, postures; while for women it was expected: perfection of femininity, dance, and rhythmic gymnastics. Of the perpetuation of this two types of stereotypes in the physical education class drift the hypothesis of this research present what is the direct and conditional influence of the cultural practices in relation to the gender expression is reduced to analysis regarding the sex condition read in code from what is biologically attributed. As a theoretical frame work we turn to the contributions of the Queer Theory, and on the other hand, we analyze current regulations for the Argentinian education system taking special reference in the law of integral sexual education of Argentina. The field work was done during the year 2018 in two primary-level public administration schools, located in the cities of Almafuerte and Rio Tercero, of the province of Córdoba, Argentina.
\end{abstract}

\section{Keywords}

Physical education. Gender. Cultural construction. Body education.

\section{Introducción}

En esta investigación, nos propusimos analizar los desafíos de la expresión de género dentro de las clases de Educación Física con niños y niñas de nivel primario, como sujetos de derecho, social y emocional, es decir, repensar la enseñanza de la Educación Física en clave de género. De ello deriva que la hipótesis que se plantea este trabajo es que: la influencia directa y condicionada de las prácticas culturales en relación a la expresión de género en las clases de Educación Física queda reducida al análisis respecto de la condición de sexo leído en clave de lo que es biológicamente atribuido. De esa manera, el poder de los mandatos sociales opera en las clases de Educación Física como dispositivo biopolítico. A finales del siglo XVIII, vemos aparecer algo que ya no es la anatonomopolítica, sino: 
[...] lo que yo llamaría una biopolítica de la especie humana. A diferencia de la disciplina, que se dirige al cuerpo, esta nueva técnica de poder no disciplinario se aplica a la vida de los hombres e, incluso, se destina, por así decirlo, no al hombre/cuerpo sino al hombre vivo, al hombre ser viviente; en el límite, si lo prefieren, al hombre/especie. [...]. Por lo tanto, tras un primer ejercicio del poder sobre el cuerpo que se produce en el modo de individualización, tenemos un segundo ejercicio del poder que no es individualizador sino masificador, por así decirlo, que no se dirige al hombre/cuerpo sino al hombre/especie. (FOUCAULT, 2006, p. 220).

En segundo lugar, avanzaremos sobre la cuestión de género y en este marco analizar los dispositivos biopolíticos que operan sobre los cuerpos en términos de géneros y sexualidades. Se entiende por expresión de género a las formas de presentación, comportamiento o intereses y afinidades que pueden ser considerados femeninos, masculinos o la combinación de ambos. Significado que le otorga la sociedad, en relación directa al rol de género (lo apropiado para un hombre o una mujer), normas sociales o comportamentales. Lo que se "espera" de cada uno respecto a las normas y normatividades. Como marco teórico, recurrimos a los aportes de la Teoría Queer y al trabajo que realiza Michel Foucault, en particular en su tercer momento.

\footnotetext{
En este artículo trabajamos con la idea propuesta por un gran amigo de Foucault, el filósofo Gilles Deleuze. Para ese autor, en la obra foucaultiana podemos identificar tres dimensiones, que responden a las crisis que Foucault atravesó en su vida: la del saber, la del poder y la de la subjetivación. Así, esas tres dimensiones '[...] constituyen una manera de vivir, una figura extraña en tres dimensiones, así como la mayor filosofía moderna [...]' (p.121). Esas tres dimensiones constituyen la tríada de la problematización de la subjetividad en Foucault. (PICH; RODRÍGUEZ, 2014, p. 465).
}

Es en este momento donde nuestro autor desarrolla el concepto de focos de experiencia como eje articulador de los tres conceptos, los desplazamientos que emprende entonces son en relación al conocimiento, al poder y al sujeto, proponiendo el análisis histórico de las formas de veridicción; el análisis histórico de los procedimientos de gubernamentalidad; y el análisis histórico de la pragmática de sí y las formas adoptadas por ella: esas eran las tres vías de acceso mediante las cuales intentó circunscribir un poco la posibilidad de una historia de lo que podríamos llamar "experiencias".

Es preciso destacar su aporte en relación también a dominios como la locura, la sexualidad, la delincuencia, etc. a partir de la vinculación que nuestro autor denomina juegos de verdad y como en las prácticas humanas el sujeto es afectado por ellos. Cuestiones referentes, por ejemplo, a categorías tales como verdadero, normal, 
aceptado precisan ser interrogadas en base al método foucaultiano, es decir ¿cómo llegamos a pensar lo que pensamos? ¿De qué manera esa noción se constituye como verdadera? ¿Por qué asociamos las prácticas corporales, el uso del cuerpo y las teorías de los gustos en relación con el sexo biológicamente atribuido?

\begin{abstract}
Actualmente, los desarrollos de Foucault se encuentran en la base de la proliferación de la teoría queer. Dicha teoría ha desestabilizado la categoría sexo a partir de las fuertes críticas esgrimidas contra la noción de identidad y naturaleza (Butler, 1999; Nouzeilles, 2002). La tendencia actual de entender las identidades en términos fragmentarios arrastra hacia el debate a la clásica distinción sexo/género. (MARTíNEZ, 2016, p. 77).
\end{abstract}

En este sentido, nos interesa destacar cómo el sujeto es afectado por los discursos "verdaderos" y de qué manera se le otorgan ese estatus y cómo es entonces que los desarrollos de la Teoría Queer nos aportan una constelación conceptual para analizar nuestro objeto.

\begin{abstract}
Queer puede ser traducido como extraño, torcido o raro. Su uso ha servido como un insulto denigrante para señalar a quienes son identificados fuera de los alcances de la sexualidad hegemónica y normativa. Sin embargo, el término fue resignificado y apropiado en un sentido positivo, de tal modo que la posición marginalizada y excluida que designa lo queer deja de ser una localización indeseada. (MARTíNEZ, 2018, p. 177-178).
\end{abstract}

La positividad de la noción en parte no solo resignifica el concepto, sino que opera como eje articulador de una infinidad de preguntas respecto a las condiciones materiales, usos y costumbres. La investigación que presentamos en este marco se encuentra atravesada por las tradiciones no solo culturales, sino que se hallan en la conformación del campo disciplinar. Habría que ver aquí la ligazón de la Educación Física con el campo deportivo, gímnico, fitnes, etc.

Así, las prácticas culturales han construido dos géneros tomando las matrices biológicamente determinadas. A partir de los movimientos y la teoría feminista, comienzan a contemplarse otras realidades, donde podemos ver más allá de lo que la sociedad ha construido de nosotros y nuestro cuerpo. Estos sentidos son inculcados desde niños y niñas, siendo interpelados por las prácticas sociales en general y por las investigaciones que desarrollamos y que se vienen desarrollando en el campo y en campos afines.

Esto implica una interpelación específica a los órdenes institucionales que se arrogan el saber de los cuerpos, las identidades y la subjetividad; especialmente el médico, pero también el jurídico. Colectivos afectados por las definiciones

Educação \& Formação, Fortaleza, v. 5, n. 14, p. 3-16, maio/ago. 2020

DOI: https://doi.org/10.25053/redufor.v5i14mai/ago.2336

http://seer.uece.br/redufor 
normativas del género y de la sexualidad van a reivindicar la disidencia a la norma antes que una liberal asunción de la diversidad. (CAMPAGNOLI, 2018, p. 4 apud FERRARI; RODRÍGUEZ, 2018, p. 2).

Para intentar arrojar luz sobre esta hipótesis, realizamos un trabajo de campo en las Ciudades de Río Tercero y Almafuerte, provincia de Córdoba (Argentina), con niños y niñas de entre 9 y 11 años. Partiendo de los aportes de investigaciones de género, sociedad y educación ante lo diverso, lo que no es "normal" o "natural", de acuerdo a estereotipos sociales, realizamos un trabajo denso de campo que nos permitió elaborar categorías sociales y analíticas. En este sentido, avanzamos sobre una visión de género más inclusiva, que toma al género, como una categoría de identidad, independientemente al sexo biológico.

El empoderamiento de los "cuerpos desviados" nos permitió incluir categorías respecto del sujeto en tanto va creando performances. En ese sentido, pusimos en consideración la posibilidad de desvanecer al sexo de la esencia biológicamente atribuida.

\begin{abstract}
El condicionamiento del sexo biológico atravesando el 'ser', con la creación de un discurso unidireccional, universal y binario [se deriva de] una normativa impuesta, donde género es asociado directamente al sexo biológico y una normativa de constructo social, de roles, relaciones (de diferencias y desigualdades entre hombres y mujeres) de poder y sometimiento, e identidad, la percepción subjetiva que un individuo tiene sobre si, en cuanto a su género, atravesada y reconstruida por componentes sociales como el rol de género, estereotipos y expresión de género. (BUSTOS, 2018, p. 4).
\end{abstract}

Esta referencia es parte de una investigación mayor que con Bustos (2018) realizamos durante los años 2017 y 2018 y que en parte los datos construidos nos permiten hoy analizar la enseñanza de la educación del cuerpo atravesada por las claves biopolíticas.

\title{
2 Materiales y métodos
}

En relación a la investigación, tomamos los aportes de Piovani (2007, p. 72), cuando afirma:

La definimos [a la investigación] [...] como un proceso que involucra un conjunto
de decisiones y prácticas (que a su vez conllevan la puesta en juego de
instrumentos conceptuales y operativos) por los cuales conocemos - lo que
puede significar describir, analizar, explicar, comprender o interpretar - algunas

Educação \& Formação, Fortaleza, v. 5, n. 14, p. 3-16, maio/ago. 2020

DOI: https://doi.org/10.25053/redufor.v5i14mai/ago.2336

http://seer.uece.br/redufor

ISSN: 2448-3583

(c) (i) Esta obra está licenciada com uma Licença Creative Commons

Atribuição 4.0 Internacional. 
situaciones de interés cuya definición y delimitación (o construcción) forma parte de las decisiones apenas aludidas.

En este marco se realizó un trabajo de pesquisa sobre la expresión de género en las clases de Educación Física utilizando métodos cuantitativos y cualitativos, operando con diversas técnicas de investigación, tales como: Focus Group, entrevistas, observaciones de campo, encuestas.

El trabajo se desarrolló con dos cursos: cuarto y quinto grados, con alumnos y alumnas de entre 9 y 11 años de dos escuelas de nivel primario de administración pública situadas en las ciudades de Almafuerte y Rio Tercero, de la provincia de Córdoba, durante el año 2018, con distintos contextos socioculturales.

El condicionamiento del sexo biológico atravesando el "ser", con la creación de un discurso unidireccional, universal y binario, se discutió a la luz de los discursos de los actores y nos permitió elaborar categorías de análisis. Los datos obtenidos se triangularon usando además como fuentes primarias las normativas vigentes para la educación básica de gestión pública.

\section{Resultados y discusión}

En primer lugar, las categorías que se tomaron para las encuestas como referencia fueron: deportes, juguetes y colores. En ese marco, se realizó una encuesta que fue administrada a 14 nenes y 11 nenas, número que también estuvo sujeto por el interés de participación y las autorizaciones legales correspondientes, dado que se trata de menores de edad. Los tres conceptos, de acuerdo a la clasificación que les otorgan las prácticas culturales como dispositivos biopolíticos, representan límites definidos para niños, para niñas, o "apto" para ambos. Evidenciando el descarte a la perspectiva de los deportes, los juguetes y colores no tienen género, una arraigada idea patriarcal mantiene el control en los patios de las escuelas, en el divertimento y preferencias de niños y niñas.

En el caso de los deportes que NO practicarían, hubo una coincidencia mayoritaria, al justificar sus elecciones asignándole determinado género, al igual que los colores. "No me gusta el rosa porque es de nena"; "No me gusta el fútbol porque es de varón". Acompañando su explicación, con el impedimento a acceder a determinada opción, por mandatos culturales, en su gran mayoría por su núcleo primitivo: la familia. 
Cabe señalar que las niñas no asociaron los colores a un género determinado. Sí, los niños en su mayoría.

En la categoría juguetes, no se manifestaron grandes porcentajes de respuestas asociadas al género. Las elecciones se basaban en la madurez que el participante posee para su manipulación, el propio divertimento que genera y la necesidad de entretenimientos tecnológicos para disfrutar del jugar.

Respecto a entrevistas, observaciones de campo y Focus Group, hemos podido interpretar que existen una normativa impuesta, donde género es asociado directamente al sexo biológicamente atribuido, y una normativa de constructo social, de roles, de relaciones (de diferencias y desigualdades entre hombres y mujeres) de poder y sometimiento e de identidad, siendo la percepción subjetiva que un individuo tiene sobre si en cuanto a su género, atravesada y reconstruida por componentes sociales como el rol de género, estereotipos y expresión de género.

Hay una concentración enérgica de la ejecución automática de contenidos, habilidades y destrezas y un desmerecimiento a la construcción de la autonomía y autoestima a través de las interacciones y connotaciones que jerarquizan (que exigen) la masculinidad, tomando con "normalidad" que la autoridad sea el sexo masculino y que la mujer se mantenga en la posición de subordinación y sumisión. Estigmatización de la práctica deportiva femenina. Estereotipos, prejuicios, funciones sociales por pertenecer al grupo social masculino o femenino, limitando la capacidad para desarrollar sus capacidades personales (Normativa: fútbol-varón, vóley-mujer). Biopolítica del deporte.

Las prácticas, movilizadas por las emociones, donde expresan lo que sienten, manifiestan las ataduras que les han construido los mandatos culturales. Las regularidades de los usos del cuerpo producen y reproducen la inquietud y desigualdad. Estigmatizados por la expresión de género en su espontaneidad: los estereotipos de masculinización con los que se presiona al sexo masculino.

Respecto a las relaciones entre pares (estudiantes) en el desarrollo de las clases de Educación Física (práctica), se pudo identificar que entre ambos sexos la dominancia es del sexo masculino sobre el femenino. Los niños identifican como sexo débil a las niñas y el sexo fuerte ellos mismos, lo cual refleja estereotipos de feminidades y masculinidades. Hay una sumisión del sexo femenino ante la normativa masculina impuesta. A nivel discursivo y de construcción a través del debate y el pensamiento, 
tomando como categorías los deportes, los juguetes y los colores, pudimos identificar también que el estereotipo más evidenciado fue la asociación de los deportes a un género determinado, por ejemplo: fútbol, masculinidad. Los niños y niñas atribuyen las diferencias y desigualdades a las influencias culturales de su núcleo familiar en primer lugar y de la escuela en segundo lugar. Manifiestan un problema en común de la obstaculización de los deseos de los sujetos (como elegir un deporte) por mandatos culturales por parte de su núcleo familiar.

Por otra parte, los colores se presentaron como el segundo estereotipo, de acuerdo a la relevancia que le significaron los niños/as por asociarlo a un determinado género. El rosa es de nenas, el azul es de varones. En cuanto al lenguaje, utilizan términos con connotaciones negativas: "marimacho", "maricón" (que nos remonta a los inicios de la Teoría Queer) de acuerdo al deporte que prefieren, a los comportamientos y maneras de expresarse. Los discursos (Focus Group) son inclusivos, pero las prácticas siguen dominadas por el universal binarismo.

La teoría queer ha sido influida por los aportes del posestructuralismo y del psicoanálisis que se refieren a la identidad, la sexualidad y al papel de lo simbólico en la construcción de las mismas. Los primeros trabajos en ella estuvieron influenciados por la obra de Michel Foucault (2008a, 2008b), Judith Butler (2007), Eve Kosofsky Sedgwick (1998) y Gayle Rubin (1989). La atención se focalizó en la deconstrucción, desmontaje y desafío de la heterosexualidad hegemónica que impregna los arreglos culturales actuales y articula todo el espectro de las expresiones y deseos sexuales, e incluye las identidades sexuales dominantes y marginadas. (MARTíNEZ, 2018, p. 179).

Esa teoría operó también en el campo educativo y como mencionamos se operativizó en el desarrollo de disposiciones, normativas y leyes que en el cotidiano escolar irrumpen y tensionan con tradiciones operantes. Por esta razón las entrevistas a profesores y profesoras, por un lado, intentaron indagar las representaciones, pero fundamentalmente buscar información respecto a la aplicación de la Educación Sexual Integral (ESI).

La Ley Nacional 26.150 (ESI) fue sancionada en Argentina el 4 de octubre del 2006, la cual establece:

[...] todos los educandos tienen derecho a recibir educación sexual integral de los establecimientos educativos públicos, de gestión estatal y privada de las jurisdicciones nacional, provincial, de la Ciudad Autónoma de Buenos Aires y municipal. Creación y objetivos de dicho programa. 
La referida ley se destina a estudiantes desde nivel inicial hasta el nivel superior de formación docente y educación técnica no universitarias (artículo 4ํำ). Adhiere además, en el artículo 1ํㅜ a la articulación de aspectos biológicos, psicológicos, sociales, éticos y afectivos. Podemos evidenciar una visión respecto al sujeto de manera totalizadora, que desarrolla en las experiencias y vivencias una construcción de dichos aspectos detallados anteriormente, desde el conocimiento, atravesados en la ejecución dentro de las instituciones educativas, por el poder que administra los contenidos, hasta el desconocimiento y acuerdos heteronormativos (únicamente) hegemónicos dualistas. Lo cual atenta con el abordaje integral de la ley, que apunta más allá de una mirada biologicista.

Contemplando la edad de los estudiantes, se proponen contenidos que permitan una comprensión más inclusiva y respetuosa de la sociedad y la convivencia de los sujetos y pares. Algunos de esos contenidos son: las distintas formas de organización, el respeto a la intimidad propia y ajena, la prevención del abuso sexual, las relaciones de género entre varones y mujeres, el respeto por la diversidad de identidades y la necesidad de luchar contra las discriminaciones y los estereotipos, entre otros; la igualdad en un sentido político.

El recorte de contenidos, acuerdos o limitaciones que docentes deben hacer con padres y madres para poder cumplimentar dicha Ley, a su vez, dejan en el camino la responsabilidad que tenemos sobre los estudiantes, y que destaca el artículo 1ํㅡ, en la continuidad y cumplimiento de aquellas leyes que manifiesten protección a través de la educación, como la Ley 25.673, de creación del Programa Nacional de Salud Sexual y Procreación Responsable, Ley 23.849, de Ratificación de la Convención sobre la Eliminación de todas las formas de Discriminación contra la Mujer, que cuentan con rango institucional, Ley 26.061, de Protección Integral de los Derechos de las Niñas, Niños y Adolescentes, y las leyes generales de educación de la Nación. Además de neutralizar gran parte de los objetivos propuestos desarrollados en el artículo 3ํ, lo cual deja una "verdad" y un conocimiento que no pueden transmitirse; o, por así decirlo, se transmite una "verdad" subjetiva, lo que no garantizaría la obligatoriedad que se encuentra sujeto al artículo 5ํำ de ESI.

Se destacan las coincidencias de las entrevistadas en referencia a las posibilidades y limitaciones para abordarla. Muestran un reconocimiento de la 
importancia del abordaje de la ESI en las escuelas y reconocen que ejecutar los contenidos es un tema tabú, por lo cual deben realizar previos consensos entre docentes y el núcleo familiar para abordar dichos contenidos (desde una mirada dualista: varón/mujer) que los resguarda de futuros inconvenientes o malestar social. Los mandatos culturales generan resistencia a los progresos de inclusión e igualdad social, respeto y valoración hacia lo diferente. A partir del ingreso de la ESI a los establecimientos educativos, se generó un movimiento social de un sector de la comunidad que marcha bajo el slogan de "Con mis hijos no te metas", donde se plantea a una infancia como propiedad de las familias, donde les niegan información seleccionada y aprobada por un equipo de profesionales idóneos para la elaboración de la ley. Desde la educación, se intenta poner en práctica un rol pedagógico con el núcleo familiar, pero, bajo éste discurso de repartición estricta de contenidos a los que se ven obligados los docentes, se mantiene al margen también a aquellos que receptan y confían en la integridad de ésta Ley y el ejercicio de la misma.

Al trabajar con grupos mixtos, en ambos establecimientos educativos, se pueden observar, dentro del curriculum oculto, las relaciones de género entre varones y mujeres, ya que las actividades se realizan de manera colectiva. El trabajo de los docentes es arduo, ya que los niños y niñas, sobre todo los niños, llevan consigo prejuicios, estereotipos y mandatos culturales que trasladan a las clases de Educación Física, que, sin el acompañamiento académico del núcleo familiar, son temas abordados con mucha tensión por parte de las instituciones educativas.

\section{Conclusiones}

Mediante esta investigación, pudimos reafirmar y comprobar nuestra hipótesis: la influencia de las prácticas culturales en la expresión de género es regulativa. Se manifiesta, como mencionamos antes, una intención discursiva de evolución, pero en el campo de las prácticas demuestra que aún hay mucho por hacer. Como actores influenciables, se mencionan a docentes; en menores casos, los pares de los niños y niñas; y, como principal detonante, el núcleo familiar, donde los sujetos construyen sus realidades con sus estereotipos y prejuicios, de lo que entienden o interpretan como "normal" para depositarlo en lugares comunes de interacción como la escuela o el patio 
de Educación Física. ¿Aquellas escuelas que no admitan este mandato cultural serán las futuras "Queer School'? Podrían entenderse como las "escuelas del margen", que se permiten el intento, riesgoso, de poner en práctica y hacer uso del derecho a una educación sexual integral, que se encuentra en permanente vigilancia por la presión social y los mandatos culturales del binarismo y la heteronorma, además de observarse en la práctica de campo los calificativos cargados de connotaciones negativas a aquellos que no se encuentren dentro de los acuerdos, un tanto arbitrarios, de lo que se espera de acuerdo a su sexo biológico, en lo que respecta a comportamientos, manera de hablar, preferencias, expresión de género.

Dentro de las escuelas, hay un discurso premeditado, con intenciones de evolución, que no condice con ideas (implantadas) y prácticas. La sociedad está en una permanente vigilancia, en la que el otro debe mantenerse en el "examen de la vida", que demuestre o reprima expresiones del "ser" para permanecer en una conducta apropiada, adecuada, "normal"2. Cuando problematizamos al sujeto, se observa una preminencia en un discurso sobre el ser varón o mujer, cuestión que no se identifica con enunciados discursivos.

Finalmente creemos que esta investigación puede ampliarse a muestras mayores, así como también desarrollarse en otros contextos sociales para poder establecer líneas comunes y diferenciadas entorno a los estereotipos que subyacen las clases de Educación Física y principalmente el lugar de los docentes frente a los grupos de alumnos y alumnas. Es desde este lugar que nuestra propuesta interpela la enseñanza de la Educación Física tradicional que se encuentra subsumida a teorías que operan desde el discurso de las ciencias biológicas y desde ahí construye discursos sobre el cuerpo y las prácticas corporales que encuentran sus limites en su propia matriz constitutiva. Nuestro desafío es contribuir a una educación del cuerpo que tome como referencia otros constructos teóricos, tales como los aportes que señalamos a los que podemos agregar las investigaciones realizadas por Michel Foucault, sobre todo en su tercer momento.

Entendemos que la última dimensión foucaultiana nos presenta valiosas herramientas teóricas para cuestionar la tradición educacional, porque el

2 "El examen es, en la actual terminología pedagógica, formativa, diseñada para que lleve una conducta prudente" (BALL, 1993, p. 43).

Educação \& Formação, Fortaleza, v. 5, n. 14, p. 3-16, maio/ago. 2020

DOI: https://doi.org/10.25053/redufor.v5i14mai/ago.2336 
problema de la verdad se sitúa en otro lugar. [...] Ese aspecto nos remite a la relación pedagógica de la escuela moderna [...] y que presupone al profesor/a como un sujeto que media el desarrollo/apropiación/construcción/movilización de operaciones cognitivas, y que pasa a ser visto como un sujeto que orienta y conduce el proceso de creación ética de sí por parte del sujeto aprendiz, y que guarda con él una relación de compromiso e implicación. (PICH; RODRÍGUEZ, 2014, p. 465).

En resumen, la investigación realizada nos permite, por un lado, analizar cómo las cuestiones discursivas operan en la dinámica escolar, siendo más dificultosas de visualizar en las prácticas corporales. Por esa cuestión, los aportes de Foucault respecto de la ética de sí nos permiten también suponer al "maestro del cuerpo" como un mediador que habilita la creación de una ética de si que en tanto ligada a formas de gobierno puede, sin embargo, revelarse, pudiendo operar con teorías que vayan más allá de las regulaciones impuestas por el discurso biológico que reduce al cuerpo a una sustancia material.

\section{Referencias}

AA.VV. Ley de Educación Sexual Integral no 26.150. Honorable Congreso de la Nación Argentina, 4 oct. 2006.

BALL, S. J. Foucault y la educación. Disciplinas del saber. Madrid: Morata, 1993.

BUSTOS, L. Entre el rosa y el azul: desafíos de la expresión de género en las clases de Educación Física. In: CONGRESO ARGENTINO DE EDUCACIÓN FÍSICA Y CIENCIAS, 13., 2019, Ensenada. Actas... Ensenada: Universidad Nacional de La Plata, 2019. Disponible en: http://sedici.unlp.edu.ar/handle/10915/86617. Aceso el: 20 mar. 2020.

BUTLER, J. El género en disputa. Barcelona: Paidós, 2007.

FEMENÍAS, M. L. Trascender. Bases teóricas en Derechos Humanos. Estado y Perspectiva de Género. 2017. Disponible en: https://www.youtube.com/watch?v=kcv keh9_imw. Aceso el: 10 oct. 2019.

FERRARI, C.; RODRÍGUEZ, N. La Educación del cuerpo en la escuela: una lectura en clave de género. In: CONGRESO ARGENTINO DE EDUCACIÓN FíSICA Y CIENCIAS, 13., 2019, Ensenada. Actas... Ensenada: Universidad Nacional de La Plata, 2019. Disponible en: http://congresoeducacionfisica.fahce.unlp.edu.ar/13oca-y-8o-laefyc/actas/mesa15_ferrari_\%20la\%20educacion.pdf/view. Aceso el: 20 mar. 2020.

FOUCAULT, M. Defender la sociedad: curso en el Collège de France (1975-1976). Buenos Aires: Fondo de Cultura Económica, 2006. 
FOUCAULT, M. El gobierno de sí y de los otros: curso en el Collège de France. Buenos Aires: Fondo de Cultura Económica, 2009.

IRIGARAY, L. Ese sexo que no es uno. Madrid: Akal. 2009.

MARTíNEZ, A. Cuerpo y poder. Impacto del pensamiento de Michel Foucault en el feminismo norteamericano de finales del Siglo XX. In: RODRíGUEZ, N. B.; VIAFARA SANDOVAL, H. (Org.). Michel Foucault, treinta años después: aportes para pensar el problema del cuerpo y la educación. La Plata: Universidad Nacional de La Plata, 2016. p. $76-86$.

MARTÍNEZ, A. Identidad y cuerpo en la trama del sujeto sexo-generizado: del psicoanálisis norteamericano a Judith Butler. La Plata: Universidad Nacional de La Plata, 2018.

MELICCI, A. L'invenzione del presente. Bolonia: I1 Mulino. 1982.

PICH, S.; RODRíGUEZ, N. Los cuerpos de Foucault: una genealogía de los estudios foucaultianos en el campo de académico de la Educación Física en Brasil y en la Argentina. Revista Brasileira de Educação Física e Esporte, São Paulo, v. 28, n. 3, p. 453-467, 2014. Disponible en: http://www.scielo.br/scielo.php?pid=S180755092014000300453\&script=sci_abstract\&tIng=es. Aceso el: 20 mar. 2020.

PIOVANI, J. El diseño de la investigación. In: MARRADI, A.; ARCHENTI, N.; PIOVANI, J. (Org.). Metodología de las ciencias sociales. Buenos Aires: Emecé, 2007. p. 71-85.

\begin{tabular}{|l|} 
Norma Beatriz Rodríguez (Buenos Aires, Argentina) \\
Universidad Nacional de La Plata, Departamento de Educación Física - Facultad de \\
Humanidades y Ciencias de la Educación, Teoría de la Educación Física \\
Magíster en Educación Corporal por la UNLP. Profesora titular de Teoría de la Educación Física 4, \\
Departamento de Educación Física, Centro Interdisciplinario Cuerpo Educación y Sociedad (Cices). \\
Instituto de Investigaciones en Humanidades y Ciencias Sociales. \\
Contribución de autoría: Colaboró con las discusiones sobre los estudios sobre la educación del \\
cuerpo en relación con la disputa de género y particularmente en las teorías feministas. Discutió cómo \\
el trabajo es parte de una investigación más amplia sobre educación corporal en la escuela. \\
E-mail: nbrodri@gmail.com.
\end{tabular}

\section{Liliana Bustos (Buenos Aires, Argentina) \\ Escuela Remedios de Escalada de San Martín, Facultad de Humanidades y Ciencias de la Educación}

Licenciada en Educación Física por la UNLP, Departamento de Educación Física, Facultad de Humanidades y Ciencias de la Educación. Profesora de la Escuela Remedios de Escalada de San Martín, Almafuerte, Argentina.

Contribución de autoría: Responsable de la contribución relacionada con el trabajo de campo, elaboración de categorías sociales y analíticas. Discutió cómo se producen las comprensiones dentro del alcance de la teoría queer y las contribuciones de las epistemologías feministas.

E-mail: lilo.palito@gmail.com. 
Editor responsable: Alfrâncio Ferreira Dias

Pareceristas ad hoc: Cristine Brandenburg e Antonio Luiz de Oliveira Barreto

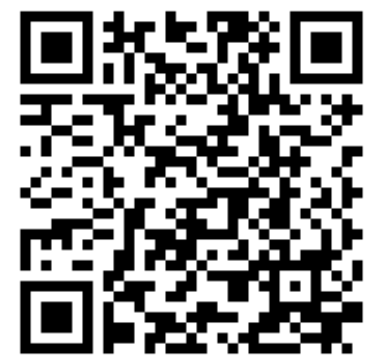

Recibido el 06 de enero de 2020.

Aceptado el 23 de enero de 2020. 